\title{
The Effect of Kinesio Taping in Reducing Myofascial Pain Syndrome on the Upper Trapezius Muscle: A Systematic Review and Meta-Analysis
}

\author{
Mansour Alotaibi \\ Department of Physical therapy, Faculty of Applied Medical Sciences, \\ Northern Border University, Kingdom of Saudi Arabia (KSA)

\section{Abdulraouf Ayoub}

Department of Physical therapy, Faculty of Applied Medical Sciences, Jazan University, Jazan, Kingdom of Saudi Arabia (KSA)

\section{Theodore King}

Department of Rehabilitation Science, College of Health Professions, concoedia, University, wisconsin, United State of America(USA)

\section{Shadab Uddin}

Department of Physical therapy, Faculty of Applied Medical Sciences, Jazan University, Jazan, Kingdom of Saudi Arabia (KSA)

\section{Doi: 10.19044/esj.2018.v14n6p336 URL:http://dx.doi.org/10.19044/esj.2018.v14n6p336}

\section{Abstract}

Background: Myofascial Pain Syndrome is a condition causing pain at myofascial trigger points. Kinesio Taping has been widely used to decrease pain and improve range of motion. Objective: The purpose of this systematic review and meta-analysis was to evaluate the effectiveness of therapeutic programs that include kinesio taping on reducing myofascial pain syndrome symptoms. Methods: Independent research was performed for legit studies using the following electronic databases: PubMed, CINAHL, MEDLINE, SPORT Discus, EM base, and Cochrane Central Register of Controlled Trials, from February 2017 to March 2017. The keywords were "keniso tape myofascial" AND "taping myofascial" AND "myofascial pain Syndrome" AND "myofascial trigger points." The research resulted in 5,793 articles that eventually included 6 articles that had met the inclusion and exclusion criteria, and the data extracted data from the articles was about the pain severity, and was measured by Visual Analog Scale (VAS). Results: The collected data was pooled from the results of 256 subjects (199 females and 57 males). Using KT showed improvement but not significant statistically in three of the analyzed studies, and the remaining three studies showed a statistical significant reduction in VAS score. The overall $\mathrm{P}$ value that computed by the 
Comprehensive Meta-Analysis 2.0 software was statistically significant (P value $=0.001$ ) between the KT group and the control group. Conclusion: This systematic review and meta-analysis was performed on six studies in regarding to the efficacy of KT on the myofascial pain in the upper trapezius muscle. The meta-analysis suggests KT with other therapeutic protocols to treat myofascial pain syndrome and increase cervical range of motion as well as the functional activities.

Keywords: Myofascial Pain Syndrome, Kinesio Taping

\section{Introduction}

Myofascial pain syndrome (MPS) is a condition causing pain at myofascial trigger points (MTrPs). MPS might present independently or concomitant with other muscular conditions. ${ }^{1}$ MTrPs are supremely sensitive spots to palpation in the skeletal muscles and appear to be the most common cause of pain in clinical practice. ${ }^{2}$

MPS is deemed to be an extremely painful condition of the musculoskeletal system and different from other chronic pain conditions. Chronic pain conditions are more common in females, though both males and females are susceptible to develop MPS. ${ }^{1}$ additionally; symptoms of MTrPs include taut bands in the muscles, weakness or tenderness at the affected spot, radial or reproduced pain, limited range of motion (ROM), and/or hot and red skin. $^{3}$

Diagnosis of MPS can be confirmed via a medical history and physical examination, and is readily identified by palpation on the suspected area. MPS is a common condition characterized by either primary or secondary pain and eventually leads to muscle spasm, restricted ROM, and functional limitations. ${ }^{1,4,5}$

MPS may be either an acute or chronic condition. Pain in acute and chronic conditions is similarly characterized by a dull deep aching discomfort or pain that is difficult to be localized and broadly distributed. Also, sharp and stabbing pain may not be present with MTrPs. ${ }^{4}$ In some instances; MPS symptoms mimic those of radiculopathy. ${ }^{1,4-6}$

Treatment of MPS common includes pain control and ROM enhancement governed by medications, splints, and physical therapy approaches. Medication options include painkillers, anti-depressants, or antiepileptics. Physical therapy options include ischemic compression, stretching techniques, manual therapy techniques, dry needling, electro therapy modalities, and taping. 4

In the 1970s, a Japanese chiropractor, Kenzo Kase, developed Kinesiology Tape (KT), an adhesive tape with unique stretchable characteristics rendering it easy to be worn on the skin without restricting 
movement. KT is made from a thin elastic adhesive material that can be stretched from 120-140\% creating a dynamic flexible tape that can simulate the human skin. ${ }^{7,8}$

Theoretically, KT treatment is based on the function of lifting the skin from the soft tissue beneath which increases the space between skin and muscle and therefore encourages blood flow and lymphatic drainage. Also, applying KT on the skin may alter the excitatory functions of the central nervous system. Therefore, KT can be used to control pain and manage muscular activities as well as improve ROM (Figure 1). ${ }^{8-10}$

BEFORE

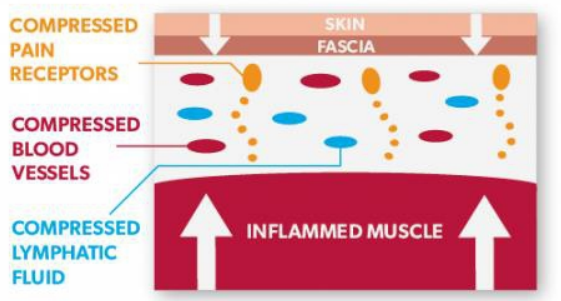

AFTER

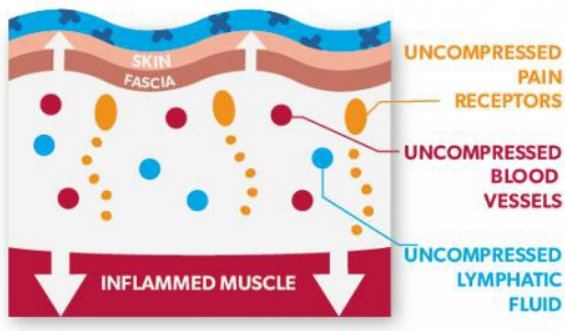

Picture from: http://themassageroom.blogspot.com/2015/12/2015-coursesattended-kinesio-taping.html

Figure 1: Illustration of the lifting effect of Kinesio Taping on the skin

KT is mostly administered in sports injuries for preventive and treatment goals creating dynamic forces which can be governed by the therapist guiding the movement towards the desired direction. KT has increasingly been used by therapists after first seen used in the Seoul Olympics in 1988. KT has evolved to be used therapeutically in a myriad of practice areas including pediatrics, neurology, osteopathy, and many other fields. ${ }^{9-12}$

$\mathrm{Wu}$, et al. ${ }^{13}$ and his colleagues reviewed the effectiveness of KT on treating myofascial pain. In their review they discussed a plethora of options in assuaging pain and other symptoms caused by MPS including isometric exercises, deep friction massage, and KT as a new method of MPS management. Additionally, they attributed origin of KT to the traditional athletic non-elastic tape. They also introduced KT application principles based on findings of previous researches.

Mense, et al. ${ }^{14}$ reported that continuous stimulation of sensory receptors from muscles leads to neuropathic alteration in the posterior horn of the spinal cord that had been excited by MTrPs pain and eventually blocks the nerve ends causing a decline in the pain level. Kase, et al. ${ }^{15}$, studied the effect of taping on blood circulation, and found that blood flow increases instantly after applying KT on the skin. 
Based on the theoretical principles of MTrPs and on empirical outcomes of KT, the authors of this study decided to investigate the efficacy of KT to alleviate MPS symptoms on the upper trapezius muscle by completing a systematic review and meta-analysis of randomized controlled trials.

\section{Methods}

This systematic review and meta-analysis was performed following the guidelines of the Preferred Reporting Items for Systematic Reviews and MetaAnalysis (PRISMA) ${ }^{16}$.

\section{Search Strategy}

Independent research was performed by the examiners from February 2017 to March 2017 for studies that matched the eligibility criteria. The research was not restricted with a specific time line. A literature search was performed using different electronic databases including PubMed, CINAHL, MEDLINE, SPORTDiscus, EMbase, and Cochrane Central Register of Controlled Trials. The keywords used for the search were "keniso tape myofascial" AND "taping myofascial" AND "myofascial pain Syndrome" AND "myofascial trigger points."

\section{Inclusion Criteria}

Randomised Controlled Trials (RCTs) of treatment protocols that included using PRP in treating chronic plantar fasciitis were eligible. RCTs were included based on the following characteristics: (1) level I of evidence, (2) sample size was higher than 20 participants, (3) English full-text articles.

\section{Exclusion Criteria}

Studies were excluded if they: (1) did not qualify as an RCT, (2) were under peer review or accepted but not published, (3) were retrospective or cohort studies, (4) were observational studies, (5) were case reports, case studies, and/or cross sectional studies, or (6) did not provide statistical analysis before and after the intervention. (7) Included other kinetic segments of MPS.

\section{Inclusion Procedure, Data Extraction, and Meta-Analysis Inclusion Procedure}

The initial search was performed by two examiners who ran the key words into the electronic databases and dismissed the redundant results. Articles that did not meet the inclusion criteria were dismissed from the search procedure. A third examiner evaluated the results and assured the search process. Also, reference lists of the chosen studies were introduced for more potential included results. 


\section{Data Extraction}

Two examiners extracted information from the marked full text articles, and all the articles were in English language. Results data extracted from the articles were pain severity, and were measured by Visual Analog Scale (VAS).

\section{Administration}

Studies were saved and analysed using MAC OS Sierra software version 10.12.3. Also, initial findings of the studies were collected and extracted via Microsoft Excel 2016 preceding logging the information into the Meta-Analysis software.

\section{Meta-Analysis}

Collected data were logged and assessed using Comprehensive MetaAnalysis 2.0 software, and the significance was considered if $P<0.05$. In case of the articles did not showed significant comparison between the intervention group (KT) and the controls, the comparison was computed by the system using t-tests based on the statistical information extracted at the base line and the last follow up. Later, statistical analysis was conducted on each article by calculating the $p$ values of the differences between groups and eventually the overall $P$ value was calculated.

\section{Risk of Bias}

The Cochrane Collaboration's Tool was used to determine the publication bias. A checklist consisting of 7-items based on box form was developed in 2005. ${ }^{17}$ The Cochrane Collaboration's Tool categorizes articles as + , low risk of bias; -, high risk of bias; and ? un clear or unknown risk of bias.

\section{Methodological Quality Measurement of Selected Research Studies}

The examiners evaluated the methodological quality of each article using the Physiotherapy Evidenced Database (PEDro) scale. The PEDro scale consists of a 10-item checklist of "Yes" or "No" questions referring to the internal validity and parametric statistical information introduced. Items were scored as either present (1) or absent (0) and a score out of 10 is figured by compuatation. ${ }^{18}$ A study scoring $\geq 7$ is considered high quality, a study scoring 5-6 is considered fair quality, and a study score $\leq 4$ is considered poor quality. ${ }^{19}$

\section{Myofascial Pain Syndrome Definitions:}

Myofascial pain syndrome (MPS) is a condition causes pain at the Myofascial Trigger Points (MTrPs) regions. MPS might present independently or concomitant with other muscular conditions. ${ }^{1}$ MTrPs are 
supremely sensitive spots to palpation in the skeletal muscles, and are appeared to be the most common inducement of pain in clinical practice. ${ }^{1,2}$

\section{Results:}

\section{Articles Stream}

The search procedure generated 5,793 articles. Following exclusion of duplicates, 68 articles were reviewed based on the title and the abstract. The remaining results after applying the inclusion criteria were 9 full-text articles. The exclusion criteria permitted 6 articles which were qualified to the current analysis. Three articles excluded; two had loss of controls and one was conducted on more than one kinetic segment. Figure 2 illustrates the schematic structure of the analysed studies using PRISMA flow charts.
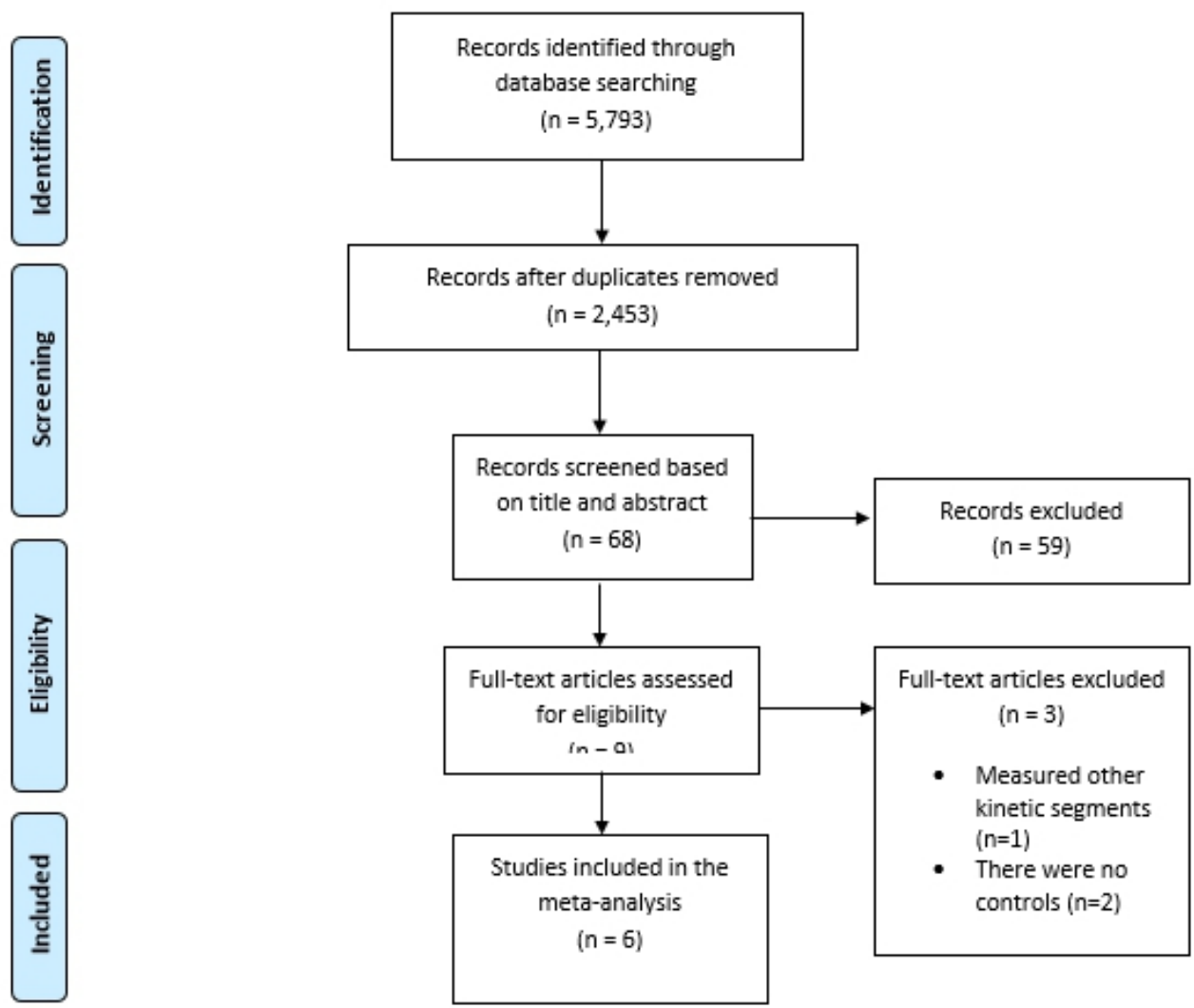

Figure 2: illustrate the schematic diagram of the articles flow and the remaining articles after applying inclusion and exclusion criteria 


\section{Baseline characteristics}

All the analyzed articles were randomized controlled trials, and level I of evidence. The demographic distribution of the articles is presented based on the chronological order as the following: one study ${ }^{20}$ was conducted in South Korea, one study ${ }^{21}$ was conducted in Poland, one study ${ }^{6}$ was conducted in Taiwan, and Three studies ${ }^{22-24}$ were conducted in Turkey. All the studies included male and female subjects with total of 256 subjects who participated in the KT group and the control group without calculating the other comparative treatment participants. Besides, female to male ratio was about 3:1 (199 females and 57 males). All Studies performed in Physical therapy department either in a university ${ }^{6,21,24}$ or in a hospital. ${ }^{20,22,23}$ Furthermore, one study $^{20}$ used KT prior to stabilization exercise twice a week for four weeks, one study ${ }^{21}$ used star shape KT primarily once for 3 days, one study ${ }^{6}$ used Yshaped KT as a primary intervention twice for 3 and four days sequentially, one study ${ }^{22}$ used inhibition application technique using I strips KT twice for three days with 1 day rest in between, one study ${ }^{23}$ used muscle technique with I strips KT in addition to stretching exercise consisting of $4 \mathrm{KT}$ sessions during the treatment period, and one study ${ }^{24}$ used I strips KT primarily five times by interval of three days .For the control group, there were three studies ${ }^{21,22,24}$ used sham tape, one study ${ }^{20}$ used stabilization exercise, one study ${ }^{6}$ used manual pressure release, and one study ${ }^{23}$ used trapezius stretching exercise. To assess the pain, all studies used Visual Analog Scale (VAS), and duration of treatment protocols escalated from 3 days to 3 months. Table 1 to summarize the baseline characteristics of the analyzed articles. 
Table 1. Baseline characteristics of the analyzed articles.

\begin{tabular}{|c|c|c|c|c|c|c|c|}
\hline Study & Year & Location & number of subjects $n=, M / F$ & Duration & Intervention & Control & Quality \\
\hline Lee et al & 2012 & $\begin{array}{l}\text { South } \\
\text { Korea }\end{array}$ & $\mathrm{n}=32,7 / 25$ & 4 weeks & Stabilization Exercise+ KT $n=16$ & Stabilization Exercise $n=16$ & $5 / 10$ \\
\hline $\begin{array}{c}\text { Halski et } \\
\text { al }\end{array}$ & 2016 & Poland & $\mathrm{n}=49,4 / 45$ & 3 days & KT $n=25$ & Sham KT n=24 & $7 / 10$ \\
\hline $\begin{array}{c}\text { Chao et } \\
\text { al }\end{array}$ & 2016 & Taiwan & $\mathrm{n}=31,7 / 24$ & 1 week & $\mathrm{MPR}+\mathrm{KT} n=16$ & $\mathrm{MPR}+\mathrm{KT} n=15$ & $8 / 10$ \\
\hline $\begin{array}{c}\text { Ozturk et } \\
\text { al }\end{array}$ & 2016 & Turkey & $\mathrm{n}=37,9 / 28$ & 1 month & $\mathrm{KT} n=20$ & Sham KT $n=17$ & $7 / 10$ \\
\hline
\end{tabular}

\section{Pain Severity Results}

Pooled data of 256 subjects, the mean VAS score for the intervention group (KT group) at the base line was 6.55 and 2.83 after the last follow up compared to the score of the control groups which was 6.13 at the baseline and 3.22 after the last follow up (Table 2).

Table 2. Illustrate the VAS values and standard deviations pre-test and post-test for KT group and the control group.

\begin{tabular}{|c|c|c|c|c|}
\cline { 2 - 5 } \multicolumn{1}{c|}{} & \multicolumn{2}{c|}{ KT Group } & Pre-test & Post-test \\
\hline Study & Pre- test & Post-test & $7.06 \pm 1.12$ & $4.75 \pm 1.69$ \\
\hline Lee. et al & $7.31 \pm 0.87$ & $4.88 \pm 1.36$ & $6.40 \pm 1.60$ & $4.90 \pm 2.20$ \\
\hline Halski. et al & $6.80 \pm 1.80$ & $5.20 \pm 2.40$ & $5.07 \pm 1.67$ & $0.73 \pm 0.88$ \\
\hline Chao. et al & $5.96 \pm 0.60$ & $0.37 \pm 0.81$ & $6.45 \pm 1.19$ & $2.60 \pm 2.82$ \\
\hline Ozturk. et al & $6.86 \pm 1.87$ & $2.64 \pm 3.25$ & $7.21 \pm 0.51$ & $2.95 \pm 0.97$ \\
\hline Azatcam. et al & $7.39 \pm 0.94$ & $1.56 \pm 0.94$ & $4.56 \pm 2.17$ & $3.93 \pm 1.96$ \\
\hline Ay et. al & $5.00 \pm 2.00$ & $2.35 \pm 1.99$ & & \\
\hline
\end{tabular}




\section{Meta-Analysis Results}

The $\mathrm{p}$ values of the comparisons between KT groups and control groups illustrated in Table 3, and eventually overall $\mathrm{p}$ value was calculated (Fig 3\&4). Studies ${ }^{20-22}$ showed remarkable reduction in VAS score which means pain had reduced compared to the control groups; however, these scores reduction were nor statistically significant $(P$ values were $0.801,0.866$, and 0.674 respectively). In contrast, the remaining three studies ${ }^{6,23,24}$ showed significant reduction in VAS that infer a significant pain alleviation in comparison to the controls ( $P$ values were $0.004,0.001$, and 0.000 respectively). Overall, $P$ value of all articles between the KT group and the control group was 0.001 which interprets the strong effect of KT on reducing MPS pain.

\section{Risk of Bias}

Cochrane Collaboration's Tool results revealed mild inequality, interpreting that the risk of bias might not be found in the analyzed articles. Table 3\&4 illustrate the results of Cochrane Collaboration's Tool and the Pedro Scale results.

\section{Meta-Analysis Forest Plot}

Study

Lee, et al. (2012) $[\mathrm{n}=32 ; \mathrm{p}=0.801]$

Halski, et al. (2016) $[\mathrm{n}=49 ; \mathrm{p}=0.866]$

Chao, et al. (2016) $[n=31 ; p=0.004]$

Ozturk, et al. (2016) $[\mathrm{n}=37 ; \mathrm{p}=0.674]$

Azatcam, et al. (2016) $[n=46 ; p=0.001]$

Ay, et al. (2017) $[n=61 ; p<0.001]$

Summary $[\mathrm{n}=256 ; \mathrm{p}<.001]$
Std diff in means and $95 \% \mathrm{CI}$

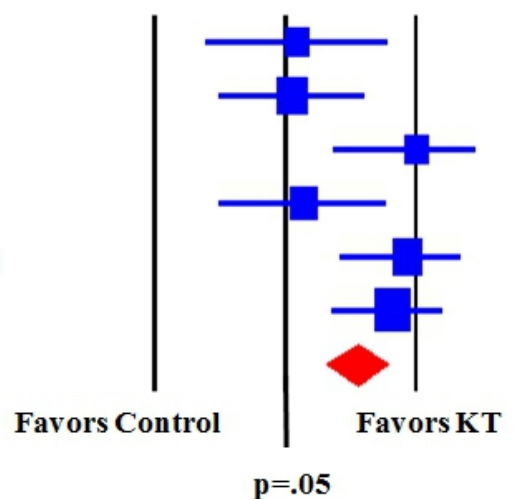

$\mathrm{p}=.05$

Figure 3: $P$ values between intervention (KT) groups and control groups and the overall $P$ value 


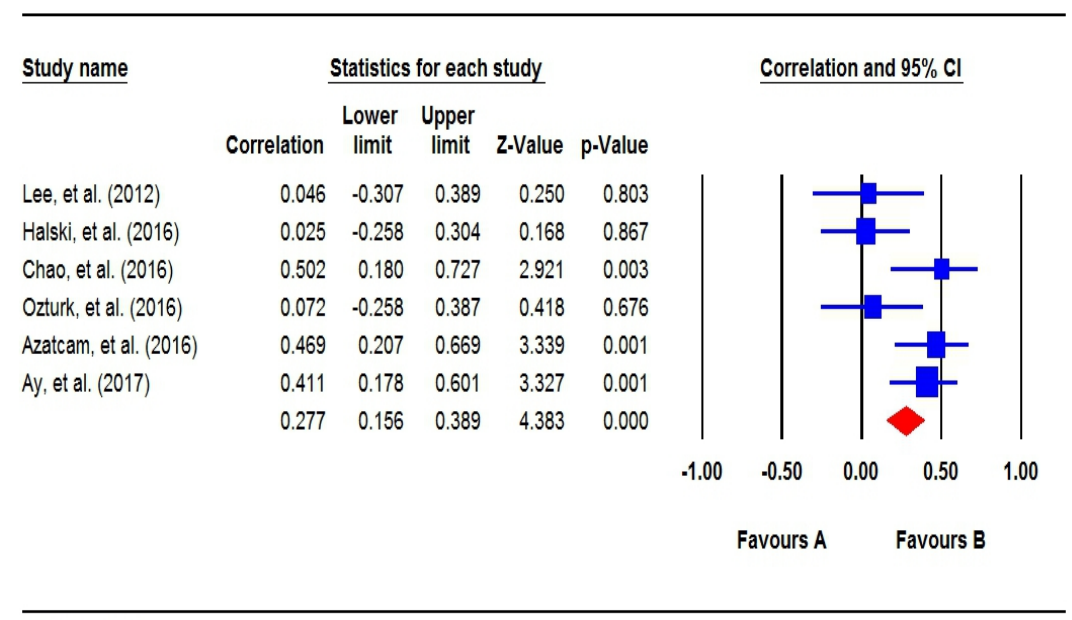

Meta Analysis

Figure 4: illustration of the statistics of the meta-analysis

Table 3: Data of Pedro Scale results:

\begin{tabular}{|c|c|c|c|c|c|c|c|c|c|c|c|c|}
\hline \multicolumn{10}{|c|}{ Table 1. Pedro scoring of analyzed studies } \\
\hline Study & 2 & 3 & 4 & 5 & 6 & 7 & 8 & 9 & 10 & 11 & $\begin{array}{c}\text { Total } \\
\text { score }\end{array}$ & Quality \\
\hline Lee et al & $\mathrm{Y}$ & $\mathrm{N}$ & $\mathrm{Y}$ & $\mathrm{N}$ & $\mathrm{N}$ & $\mathrm{N}$ & $\mathrm{Y}$ & $\mathrm{Y}$ & $\mathrm{N}$ & $\mathrm{Y}$ & $5.00 / 10.00$ & Fair \\
\hline $\begin{array}{c}\text { Halski et } \\
\text { al }\end{array}$ & $\mathrm{Y}$ & $\mathrm{N}$ & $\mathrm{Y}$ & $\mathrm{Y}$ & $\mathrm{N}$ & $\mathrm{N}$ & $\mathrm{Y}$ & $\mathrm{Y}$ & $\mathrm{Y}$ & $\mathrm{Y}$ & $7.00 / 10.00$ & High \\
\hline $\begin{array}{c}\text { Chao et } \\
\text { al }\end{array}$ & $\mathrm{Y}$ & $\mathrm{N}$ & $\mathrm{Y}$ & $\mathrm{Y}$ & $\mathrm{N}$ & $\mathrm{Y}$ & $\mathrm{Y}$ & $\mathrm{Y}$ & $\mathrm{Y}$ & $\mathrm{Y}$ & $8.00 / 10.00$ & High \\
\hline $\begin{array}{c}\text { Ozturk } \\
\text { et al }\end{array}$ & $\mathrm{Y}$ & $\mathrm{N}$ & $\mathrm{Y}$ & $\mathrm{Y}$ & $\mathrm{N}$ & $\mathrm{N}$ & $\mathrm{Y}$ & $\mathrm{Y}$ & $\mathrm{Y}$ & $\mathrm{Y}$ & $7.00 / 10.00$ & High \\
\hline $\begin{array}{c}\text { Azatcam } \\
\text { et al }\end{array}$ & $\mathrm{Y}$ & $\mathrm{N}$ & $\mathrm{Y}$ & $\mathrm{Y}$ & $\mathrm{N}$ & $\mathrm{N}$ & $\mathrm{Y}$ & $\mathrm{Y}$ & $\mathrm{Y}$ & $\mathrm{Y}$ & $7.00 / 10.00$ & High \\
\hline Ay et al & $\mathrm{Y}$ & $\mathrm{N}$ & $\mathrm{Y}$ & $\mathrm{Y}$ & $\mathrm{N}$ & $\mathrm{Y}$ & $\mathrm{N}$ & $\mathrm{Y}$ & $\mathrm{Y}$ & $\mathrm{Y}$ & $7.00 / 10.00$ & High \\
\hline $\begin{array}{c}\% \text { of Y } \\
\text { Per } \\
\text { Criterion }\end{array}$ & $100 \%$ & $0 \%$ & $100 \%$ & $83.33 \%$ & $0 \%$ & $33.33 \%$ & $83.33 \%$ & $100 \%$ & $83.33 \%$ & $100 \%$ & $\begin{array}{c}\text { Score } \\
\text { Average: } \\
6.83 / 10.00\end{array}$ & \\
\hline
\end{tabular}

Criterion Satisfied; $\mathrm{N}=$ Criterion not satisfied

2. Random allocation to groups

3. Allocation was concealed

4. Similar group at base line regarding important prognostic indicators

5. Blinding of all subjects

6. Blinding of therapist who administrated the therapy

7. Blinding of all assessors who measured at least one key outcome

8. Measure of at least one outcome for more than $85 \%$ of subjects

9. All subjects received the intervention or "intention to treat" was stated

10. Between-group statistical comparison for at least one key outcome

11.Point measures and measures of variability for at least one key outcome

Table 4. Illustration of the Cochrane Collaboration's Tool results.

\begin{tabular}{|c|c|c|c|c|c|c|c|c|}
\hline Study & 1 & 2 & 3 & 4 & 5 & 6 & 7 & 8 \\
\hline Lee et al & + & - & - & - & - & + & + & - \\
\hline
\end{tabular}




\begin{tabular}{|c|c|c|c|c|c|c|c|c|}
\hline Halski et al & + & - & + & - & - & + & + & - \\
\hline Chao et al & + & - & + & - & + & + & + & $?$ \\
\hline Ozturk et al & + & - & + & - & - & + & + & $?$ \\
\hline Azatcam & + & - & + & - & - & + & + & $?$ \\
\hline Ay et al & + & - & + & - & - & + & + & + \\
\hline \multicolumn{8}{|c|}{ (+), low risk of bias;(-), high risk of bias; (?), unknown risk of bias } \\
1. Random sequence generation (selection bias) \\
2. Allocation concealment (selection bias) \\
3. Blinding of participant \\
4. Blinding of provider \\
6. incomplete outcome data (selection bias) \\
7. Selective reporting (selection bias) \\
8. other bias
\end{tabular}

\section{Discussion}

This is the first meta-analysis that evaluated the effect of Kinesio Tape (KT) on the myofascial pain on the upper trapezius muscle area. Our study included six Randomized controlled trials ${ }^{6,20-24}$ implemented significant role of kinesio tape on assuaging myofascial pain in the upper trapezius region. Accordingly, using weather only KT or concomitant with other traditional exercise on the upper trapezius area decreases pain.

Latent Myofascial Trigger Point (MTrPs) is mostly concentrated in the upper trapezius region in the shoulder area ${ }^{25,26}$. Fischer ${ }^{27}$ measured the muscle sensitivity to pain using pressure threshold meter on seven muscles around the shoulder joint, two muscles on the lumbar region, and on muscle in the hip joint. The results of his study confirmed that upper trapezius muscle is the most sensitive muscle to pain, and females are significantly more prone to MTrPs in the upper trapezius muscle than males.

Among the analyzed studies, there was not united application of the KT. Various taping techniques have been used to measure the effect of the KT on the MPS. The taping concept can be used for many purposes including muscular inhibition, ligament application, fascial application, lymphatic applications, and etc. ${ }^{28-31}$ Besides, the tape characteristics are different among the available products. These factors might affect the results of using KT in decreasing pain; however, the proper technique should be performed after full examination to the affected muscle.

Moreover, three studies ${ }^{20,23,24}$ examined the effect of KT on pain relief and the functional activity or disability level. In contrast, the remaining three studies ${ }^{6,21,22}$ analyzed the effect of KT on reducing pain with other parameters. For instance, Chao et $\mathrm{al}^{6}$ evaluated VAS, muscle stiffness, and 
mechanomyography. However, comparing affected muscle with the sound muscle in the other side has not been done in neither analyzed study.

$\mathrm{Wu}$ et $\mathrm{al}^{28}$ and his colleagues reviewed the effect of KT on myofascial pain, and refer the mechanism of $\mathrm{KT}$ in reducing pain to the other physical therapy interventions principles. So that, they hypothesized that KT reduces MTrPs through increasing the space between the inflamed fascial, and therefore two factors play role in alleviate pain. First, blood circulation improved to discard the heat elements from inflammation. Second, the pressure on nociceptors reduced on the inflamed area.

Aleksiev $^{32}$ used distinct technique by doing taping with postisometric relaxation (PIR-taping) to evaluate its efficacy on treating myofascial pain. Interestingly, he highlighted the continuous effect of this technique because of the presence of taping. Also, he found that PIR-taping is more useful with children to achieve adequate muscle contraction. In some cases when voluntary contraction is necessary needed, PIR-taping is a recommended approach to avoid complications of those situations.

\section{Strength and Limitations}

This study included high standards randomized controlled studies and focused intervention on very specific illness. However, many restriction of this studies has been inferred after deep evaluation. Firstly, the articles declared the default KT wearing duration, but it is hard to detect the sustainability of the tape or if any peeling of the tape occurred. Secondly, using various taping technique had an effect on the comparative results of the studies. In other words, there was no formal technique to use in this specific diagnosis even though the goal was to increase space in the inflamed tissue, Thirdly, studies which used another intervention as a control (e.g., stretching exercise) contributed in the bias of measuring the true impact of the KT on the MPS. Using sham tape reveled more accurate values of the effect of the KT; however, the career ethics and adequate potential reasons for not doing these types of controls.

\section{Directions for Future Studies}

This study recommend conducting more RCTs on the effect of KT on the MPS pain in other kinematic regions to investigate its therapeutic efficacy. The basics of KT concept are relatively understandable and we can hypothesis from this study that KT can produce significant reduction in pain caused by MPS. However, to measure the accurate effect of KT in the future study, and since it easy to compare tape with sham tape, using sham tape as a control is strongly recommended. 


\section{Acknowledgment}

This systematic review was performed under the supervision of the Rehabilitation Science Department at Concordia University Wisconsin, and with a direct supervision of the head of the department Dr. Theodor King. Additionally, the main other is a current certified taping practitioner approved by the Cure Tape(C.

\section{Conclusion}

This systematic review and meta-analysis was performed on six studies in regarding to the efficacy of KT on the myofascial pain in the upper trapezius muscle. The meta-analysis confirmed the fact that using KT alone in this illness can assuage myofascial pain. In addition, Appling KT accompanied by exercises can generate greater decrease in pain and increase cervical range of motion as well as the functional activities. These results suggested that the KT is significantly efficacious in treating myofascial pain on the upper trapezius muscle with or without other therapeutic protocols.

\section{References:}

1. Borg-Stein J, Iaccarino MA. Myofascial pain syndrome treatments. Physical medicine and rehabilitation clinics of North America. 2014;25:357-374.

2. Saxena A, Chansoria M, Tomar G, Kumar A. Myofascial Pain Syndrome: An Overview. Journal of Pain \& Palliative Care Pharmacotherapy. 2015;29:16-21.

3. Lavelle W, Lavelle ED, Smith HS. Myofascial Trigger Points. Medical Clinics of North America. 2007;91:229-239.

4. Gerwin RD. Diagnosis of myofascial pain syndrome. Physical medicine and rehabilitation clinics of North America. 2014;25:341355.

5. Sharan D. Myofascial pain syndrome: Diagnosis and management. Indian Journal of Rheumatology. 2014;9:S22-S25.

6. Chao YW, Lin JJ, Yang JL, Wang WT. Kinesio taping and manual pressure release: Short-term effects in subjects with myofasical trigger point. Journal of hand therapy : official journal of the American Society of Hand Therapists. 2016;29:23-29.

7. Preece H, White P. Does kinesiology tape increase trunk forward flexion? Journal of Bodywork and Movement Therapies. 2016.

8. Lee CR, Lee DY, Jeong HS, Lee MH. The Effects of Kinesio Taping on VMO and VL EMG Activities during Stair Ascent and Descent by Persons with Patellofemoral Pain: a Preliminary Study. Journal of Physical Therapy Science. 2012;24:153-156. 
9. Williams S, Whatman C, Hume PA, Sheerin K. Kinesio Taping in Treatment and Prevention of Sports Injuries: A Meta-Analysis of the Evidence for its Effectiveness. Sports Medicine. 2012;42:153-164.

10. Thelen MD, Dauber JA, Stoneman PD. The clinical efficacy of kinesio tape for shoulder pain: a randomized, double-blinded, clinical trial. The Journal of orthopaedic and sports physical therapy. 2008;38:389-395.

11. Mezzedimi C, Livi W, Spinosi MC. Kinesio Taping in Dysphonic Patients. Journal of Voice. 2017.

12. Serra, Maysa V G B, Vieira ER, Brunt D, Goethel MF, Gonçalves M, Quemelo PRV. Kinesio Taping effects on knee extension force among soccer players. Brazilian journal of physical therapy. 2015;19:152158.

13. Wu W, Hong C, Chou L. The Kinesio Taping Method for Myofascial Pain Control. Evidence-based complementary and alternative medicine : eCAM. 2015;2015:950519.

14. Mense S. Nociception from skeletal muscle in relation to clinical muscle pain. Pain. NETHERLANDS: Elsevier B.V; 1993;54:241289.

15. D. C. K. Kase, Illustrated Kinesio Taping Manual, Kent-Kai, Tokyo, Japan, 2nd edition, 1997.

16. Moher D, Liberati A, Tetzlaff J, Altman DG, PRISMA Group. Preferred reporting items for systematic reviews and meta-analyses: the PRISMA statement. Annals of internal medicine. 2009;151:264.

17. Julian P T Higgins, Altman DG, Gøtzsche PC, et al. The Cochrane Collaboration's tool for assessing risk of bias in randomised trials. BMJ: British Medical Journal. 2011;343:889-893.

18. Maher CG, Sherrington C, Herbert RD, Moseley AM, Elkins M. Reliability of the PEDro Scale for Rating Quality of Randomized Controlled Trials. Physical Therapy. 2003;83:713.

19. Walser RF, Meserve BB, Boucher TR. The effectiveness of thoracic spine manipulation for the management of musculoskeletal conditions: a systematic review and meta-analysis of randomized clinical trials. The Journal of manual \& manipulative therapy. 2009;17:237246.

20. Lee J, Yong M, Kong B, Kim J. The Effect of Stabilization Exercises Combined with Taping Therapy on Pain and Function of Patients with Myofascial Pain Syndrome. Journal of Physical Therapy Science. 2012;24:1283-1287.

21. Halski T, Ptaszkowski K, Słupska L, et al. Short-Term Effects of Kinesio Taping and Cross Taping Application in the Treatment of Latent Upper Trapezius Trigger Points: A Prospective, Single-Blind, 
Randomized, Sham-Controlled Trial. Evidence - Based Complementary and Alternative Medicine. 2015;2015:1-9.

22. Öztürk G, Külcü DG, Mesci N, Şilte AD, Aydog E. Efficacy of kinesio tape application on pain and muscle strength in patients with myofascial pain syndrome: a placebo-controlled trial. Journal of Physical Therapy Science. 2016;28:1074-1079.

23. Azatcam G, Atalay NS, Akkaya N, et al. Comparison of Effectiveness of Transcutaneous Electrical Nerve Stimulation and Kinesio Taping added to Exercises in Patients with Myofascial Pain Syndrome. Journal of Back and Musculoskeletal Rehabilitation. 2016;13:49.

24. Ay S, Konak HE, Evick D, et al. The Effectiveness of Kinesio Taping on Pain and Disability in Cervical Myofascial Pain Syndrome. Rev Bras Reumatol. 2017;57:93-99.

25. Sciotti VM, Mittak VL, DiMarco L, et al. Clinical precision of myofascial trigger point location in the trapezius muscle. Pain. 2001;93:259-66

26. Wade R. Trigger points in the upper trapezius or normal subtrapezial anatomy? Physiother Can. 2001;53:219-22.

27. Fischer AA. Pressure algometry over normal muscles. Standard values, validity and reproducibility of pressure threshold. Pain. 1987;30:115-26.

28. Wu W, Hong C, Chou L. The Kinesio Taping Method for Myofascial Pain Control. Evidence - Based Complementary and Alternative Medicine. 2015;2015:1-9.

29. Yoshida A, Kahanov L. The Effect of Kinesio Taping on Lower Trunk Range of Motions. Research in Sports Medicine. 2007;15:103-112.

30. Kalichman L, Frenkel-Toledo S, Vered E, et al. Effect of kinesio tape application on hemiplegic shoulder pain and motor ability: a pilot study. International Journal of Rehabilitation Research. 2016;39:272276.

31. Windisch C, Brodt S, Röhner E, Matziolis G. Effects of Kinesio taping compared to arterio-venous Impulse System ${ }^{\mathrm{TM}}$ on limb swelling and skin temperature after total knee arthroplasty. International Orthopaedics. 2017;41:301-307.

32. Aleksiev AR. A novel physical therapy method of treating myofascial pain due to muscle spasm and shortening. Folia medica. 2013;55:43. 